\title{
Synthesis of a Sparse 2D-Scanning Array using Particle Swarm Optimization for Side-Lobe Reduction
}

\author{
SIVARANJAN GOSWAMI, KANDARPA KUMAR SARMA \\ Department of Electronics of Communication Engineering Gauhati University Guwahati, INDIA \\ KUMARESH SARMAH \\ Department of Electronics of Communication Technology Gauhati University Guwahati, INDIA
}

\begin{abstract}
Synthesis of sparse arrays is a promising area of research for a wide range of applications including radar and millimeter-wave wireless communication. The design goal of array thinning problems is to reduce the number of elements of an array without significantly affecting its performance. This work presents a technique for synthesizing a sparse phased-array antenna from a $16 \times 16$ uniform rectangular array (URA). The proposed approach reduces the number of elements by $50 \%$ without any significant increase in the peak sidelobe level (PSLL) for all possible scan angles in the azimuthal and elevation plans within a finite range of scan angles. The synthesis includes an artificial neural network (ANN) model for estimation of the excitation weights of the URA for a given scan-angle. The weights of the sparse array are computed by the Hadamard product of the weight matrix of the URA with a binary matrix that is obtained using particle swarm optimization (PSO) to minimize the PSLL.
\end{abstract}

Keywords - sparse array antenna; planar array; peak sidelobe level; PSLL; particle swarm optimization Received: February 14, 2021. Revised: July 19, 2021. Accepted: July 31, 2021. Published: August 10, 2021.

\section{Introduction}

A phased array antenna is widely used in wireless communication and radar systems. With the evolution of $5 \mathrm{G}$ and millimeter-wave communication, a large grid of small printed antennas is becoming popular $[1,2]$.

A phased array antenna comprises stationary elements excited at different phases to obtain radiation in different directions [3]. Phased arrays have been there for a long time. The first phased array antenna was made in 1955 [4]. The first printed phased array was reported by Munson et al. in 1974 [5]. With the evolution of microwave and millimeterwave communication standards, the use of phased arrays became more common. There has been extensive research on phased array antennas with a significant number of radiating elements for $5 \mathrm{G}$ wireless communication [6].

The elements of a typical phased array have a spacing of one-half of the operating wavelength, represented by $\lambda / 2$ [3]. A sparse array is a phased array antenna that has fewer elements than a conventional array. Synthesis of a sparse array reduces the overall cost, weight, required power, dissipated heat, etc. of a communication or a radar system because of the reduced number of elements of the array and the corresponding reduction in the excitation circuitry [7].

When an array has a fewer number of elements, the spacing between the elements becomes greater than $\lambda / 2$. This causes an increase in the number of side lobes of the antenna. This is a major drawback of sparse arrays. Traditionally, this problem was addressed by adjusting the positions, spacing, and excitation weights of the array [7].

With the advancement of modern computers, softcomputational optimization algorithms are widely being used for the synthesis of sparse arrays. Synthesizing a sparse array from a fully populated array is called an array thinning problem. A solution to an array thinning problem using genetic algorithm was proposed by R. Jain et al. in 2012 [8]. Another similar work was published by M. A. Zaman et al. in 2012 [9]. There are also analytical approaches for the synthesis of arrays. In 2016, E. Sandi et al. proposed a technique for the synthesis of sparse arrays using a combination of cyclic difference set and binomial amplitude tapering [10]. Such approaches usually involve a complex mathematical formulation and limited usability.

In recent years, the synthesis of planar sparse arrays is emerging as a popular area of research. A modified genetic algorithm for the synthesis of planar arrays was proposed in 2017 by K. Y. Reddy et al [11]. Another multi-objective optimization-based technique for sparse array synthesis was proposed in 2020 by H. Li et al [12]. In both of these works, the primary objective was to minimize the peak sidelobe power. The radiation pattern of the antenna is calculated numerically to obtain the value of the fitness function. There are also analytical approaches for the synthesis of planar phased array antennas. A singular value decomposition (SVD) based non-iterative approach for array synthesis was reported by P. F. Gu et al. in 2019 [13].

Arrays of printed antennas are most commonly used for millimeter-wave communication. Most of the printed antennas with a ground plane have a cosine radiation pattern. In this work, a sparse 2D phased array is presented with cosine antenna elements. The sparse array is synthesized from a $16 \times 16$ uniform rectangular array (URA). The number of elements in the array is reduced by $50 \%$. The positions of the elements are tuned with Particle Swarm Optimization (PSO) algorithm to minimize the peak sidelobe level (PSLL).

The remaining sections of the paper are arranged as follows. The design details of the $16 \times 16$ URA are presented in Section II. Section III covers the details of the synthesis 
and optimization of the sparse array followed by the experimental results and discussions in Section IV. The paper is concluded in Section V.

Matlab Phased Array System Toolbox ${ }^{\circledR}$ is used for computing all radiation patterns used and presented in this work.

\section{Design of a $16 \times 16$ URA \\ 2.1 Array Topology and Progressive Phase Excitation}

The topology of the uniform rectangular array is shown in Fig. 1. It is a uniform array with a spacing of half of the wavelength $(\lambda / 2)$ in both directions. The antenna element used is a cosine element.

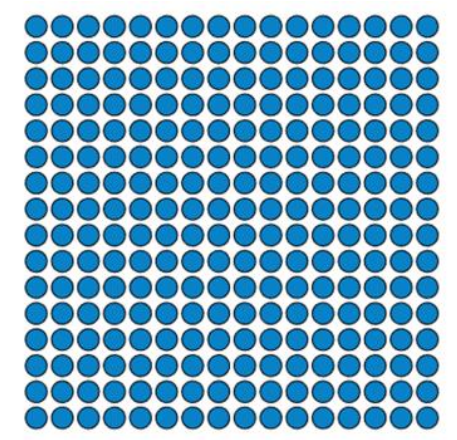

Fig. 1. Topology of the URA

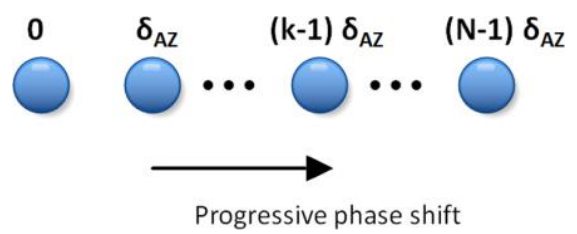

(a) Progressive phase shift in the direction of azimuthal plane

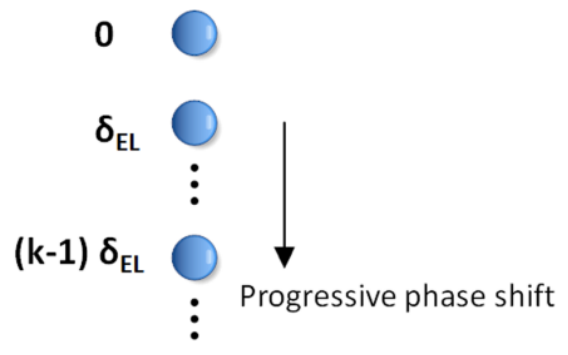

$(\mathrm{N}-1) \delta_{\mathrm{EL}}$

(b) Progressive phase shift in the direction of azimuthal plane

Fig. 2. Illustration of the progressive phase shift in excitation

The antenna elements are excited with a progressive phase difference in the directions of both azimuthal plane and elevation plane. The progressive phase shift is illustrated in Fig. 2 (a) and (b). The excitation weights of the antenna are calculated from these angles of progressive phase difference along the directions of the azimuth angle and the elevation angle.
Along the direction of the azimuth plane, the $\mathrm{k}^{\text {th }}$ element has a phase of (k-1) $\delta_{A z}$. Similarly, the $\mathrm{m}^{\text {th }}$ element along the direction of the elevation angle has a phase of (m-1) $\delta_{\mathrm{AZ}}$. Thus, the weight of excitation of the element $(k, m)$ in the $2 \mathrm{D}$ array is given by Eq. 1 .

$$
\begin{aligned}
w= & e^{j(k-1) \delta_{A Z}} \times e^{j(m-1) \delta_{E L}}=e^{j\left[(\mathrm{k}-1) \delta_{A Z}+(m-1) \delta_{E L}\right]} \\
= & \cos \left[(\mathrm{k}-1) \delta_{A Z}+(\mathrm{m}-1) \delta_{E L}\right]+ \\
& j \sin \left[(\mathrm{k}-1) \delta_{A Z}+(\mathrm{m}-1) \delta_{E L}\right]
\end{aligned}
$$

For a uniform linear array, the analytical equations are available for estimating the values of the direction of the major lobe from the value of the progressive phase shift $(\delta)$ [3]. In this work, an experimental method is used to understand this correlation for the 2D planar array. A dataset is created by varying both $\delta_{\mathrm{AZ}}$ and $\delta_{\mathrm{EL}}$ within a range of -135 degree to 135 degree at intervals of 15 degree resulting in a total of 361 scan-angles. The direction of the major lobe of the resultant radiation pattern is represented in terms of a combination of the azimuth angle $(\phi)$ and the elevation angle $(\theta)$ in a $3 \mathrm{D}$ polar coordinate system. The correlation plots are shown in Fig. 3.

The sign of the correlation depends on the choice of the coordinate system. Here, the elevation angle is positive towards the top and negative towards the bottom and therefore a positive correlation is observed. The azimuth angle, on the other hand, is positive towards the right and negative towards the left leading to a negative correlation.

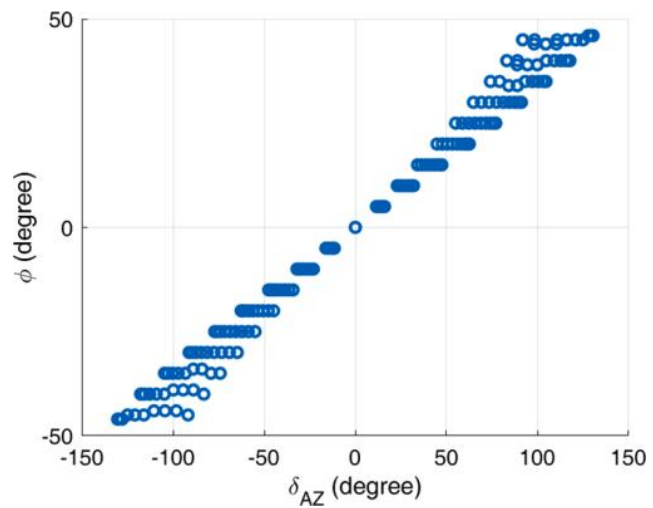

(a)

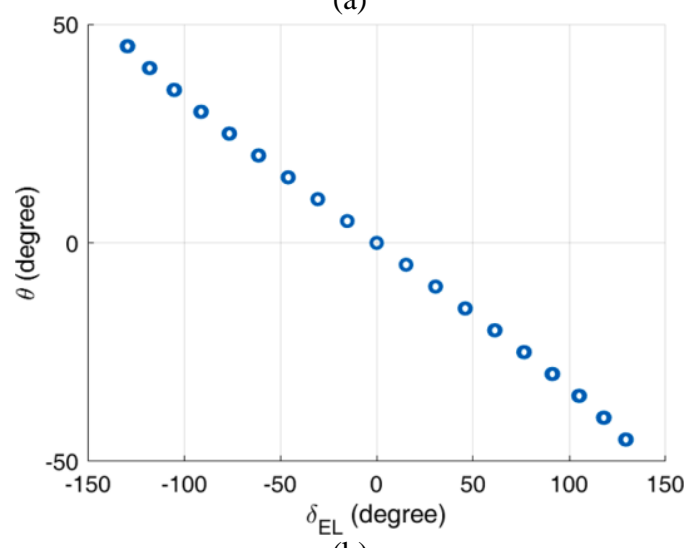

(b)

Fig. 3 Correlation of the (a) Azimuth angle $(\phi)$ with $\delta_{\mathrm{AZ}}$ and (b) Elevation angle $(\theta)$ with $\delta_{\mathrm{EL}}$ 


\subsection{Mapping the Radiation Angle to the Progressive Phase Shift using ANN}

From Fig. 3(a) it is observed as $\delta_{\mathrm{AZ}}$ and $\delta_{\mathrm{EL}}$ is varied from -135 degree to +135 degree, the corresponding values of $\phi$ and $\theta$ vary from -45 degree to +45 degree. The elevation component of the radiation pattern shows a consistent linear correlation with the value of $\delta_{\mathrm{EL}}$. However, the relation between $\theta$ and $\delta_{\mathrm{AZ}}$ is not consistent. It is evident from this observation that the value of $\theta$ depends upon both $\delta_{\mathrm{AZ}}$ and $\delta_{\mathrm{EL}}$.

For modeling such systems, computational approaches are more suitable than analytical approaches since the computational models can detect hidden patterns in the data that cannot be observed or modeled analytically [14]. An artificial neural network (ANN) model is trained to map the angle of the major lobe $(\phi, \theta)$ with the progressive phase angle $\left(\delta_{\mathrm{AZ}}, \delta_{\mathrm{EL}}\right)$. The architecture of the ANN model is shown in Fig. 4.

The ANN model is trained with the data set prepared for observing the correlation. Since the dataset is relatively small, a shallow network with 5 neurons in the hidden layer is selected for this purpose. The dataset is randomly split into test data and train data. The network is trained with a Bayesian Regularization algorithm which is suitable for smaller datasets $[15,16]$.

The error histogram of the neural network training is shown in Fig. 5. A peak error of \pm 1.15 degree is observed which is acceptable for this problem.

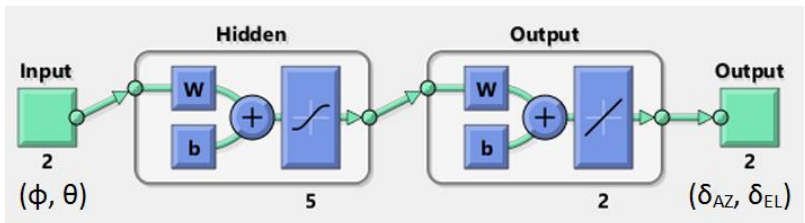

Fig. 4. Architecture of the ANN model to map the radiation angles $(\phi, \theta)$ with the progressive phase angle $\left(\delta_{\mathrm{AZ}}, \delta_{\mathrm{EL}}\right)$

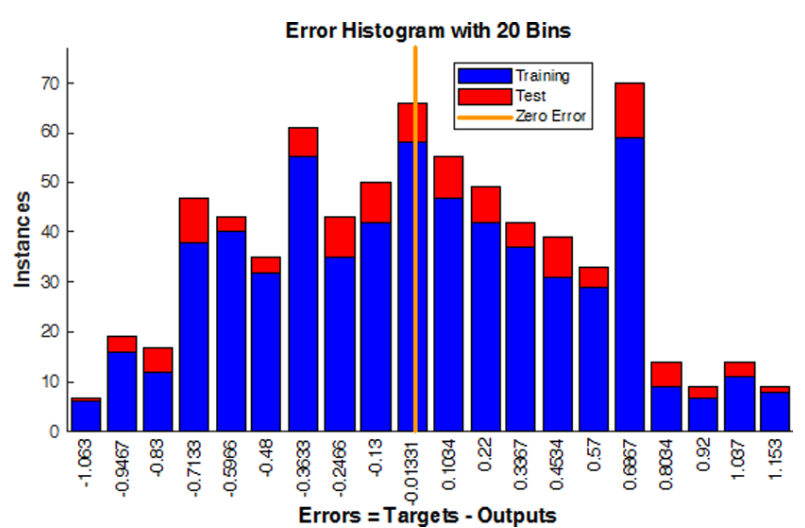

Fig. 5. Error histogram of the trained neural network

\section{Synthesis of Sparse Array}

The key challenge in synthesizing a sparse scan-array is to ensure that the PSLL is minimized for all possible scanangles or all possible combinations of $\delta_{\mathrm{AZ}}$ and $\delta_{\mathrm{EL}}$. Calculating the radiation pattern for all possible combinations is computationally very expensive. To make the experiment feasible, the radiation pattern is computed for three randomly selected $(\phi, \theta)$ pairs. For each of these pairs, the corresponding values of $\delta_{\mathrm{AZ}}$ and $\delta_{\mathrm{EL}}$ are obtained from the trained ANN model. The radiation pattern of the antenna is computed for each of these three $(\phi, \theta)$ pairs. The excitation weight matrix, $\mathrm{W}$ of the URA is calculated using Eq. 1. The objective function returns the maximum PSLL value out of the three $(\phi, \theta)$ pairs.

This step makes the objective function computationally expensive. To compensate for this, the PSO algorithm is used. The PSO is a widely used bio-inspired optimization algorithm and it is computationally less expensive than genetic algorithms (GA) as it requires fewer iterations [17].

The flowchart of the proposed approach for sparse array synthesis using PSO is shown in Fig. 6. Here, W is the excitation weight matrix of the $16 \times 16$ URA. B is a binary matrix of size $16 \times 16$. The weight of the sparse array is given by the Hadamard product of $\mathrm{B}$ and $\mathrm{W}(\mathrm{B} \odot \mathrm{W})$. Thus, the optimization problem can be defined mathematically as:

$$
\text { Minimize } F[B \odot W]
$$

Where $\mathrm{F}$ is the function that yields the maximum PSLL of the three randomly selected $(\phi, \theta)$ pairs. In order to make sure that exactly $50 \%$ of the elements are removed by the $\mathrm{PSO}$, an additional constraint is added which is given by Eq. (4).

$$
\sum_{i, j}\left[B_{i, j}\right]_{N \times N}=\frac{N}{2}
$$

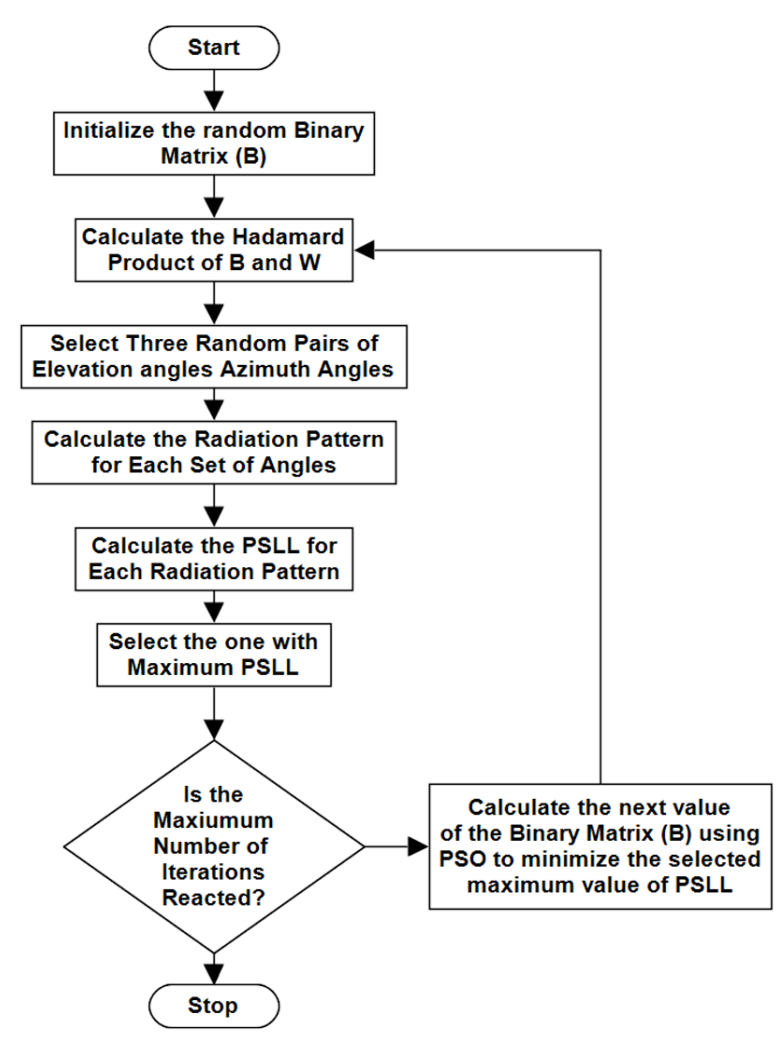

Fig. 6. Flow diagram of the sparse array synthesis steps using PSO 


\section{Experimental Results and Discussions}

The $16 \times 16$ URA is thinned into a sparse array using the method discussed in Section III. In this section, the results of various experiments performed are covered to validate the accuracy of the proposed technique.

\subsection{The Architecture of the Sparsed Array}

The element positions of the synthesized sparse array are shown in Fig. 7. Here, the number of elements in the sparse array is 128 . The original $16 \times 16$ URA has 256 elements. Thus, the number of elements in the array is reduced by $50 \%$.

It is observed that at some parts of the synthesized array, the vertical spacing of the original URA is maintained whereas, in some other parts, the horizontal spacing is maintained. This architecture guarantees that the excitation weights calculated for the URA work for the sparse array as well. Moreover, the elements that are scattered do not form any regular pattern. This suppresses the possibility of larger side-lobes that appear at multiples of the desired values of $\phi$ and $\theta$. It is difficult to obtain such solutions analytically.

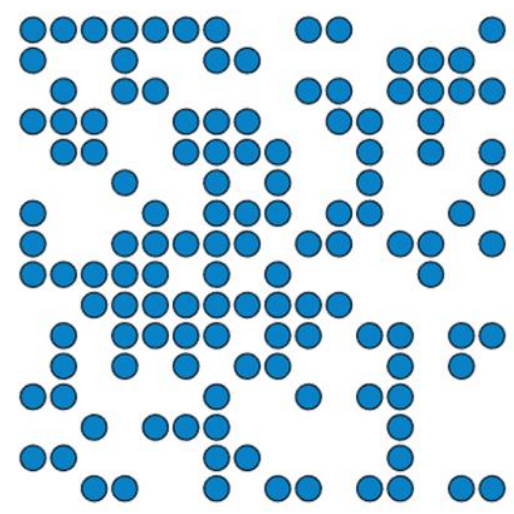

Fig. 7. Element positions of the synthesized sparse array.

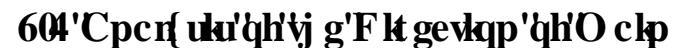 \\ 7) REHIDQGB36/ /}

The radiation patterns of the synthesized sparse array are analyzed for many combinations of the $(\phi, \theta)$ pair. A part of these results is shown in Table 1 . The table shows the required values of $\phi$ and $\theta$, obtained values of $\phi$ and $\theta$, and the PSLL values of the URA and the sparse array.

TABLE I. SOME OF THE ANGLES CONSIDERED

\begin{tabular}{|c|c|c|c|c|c|}
\hline $\begin{array}{c}\phi(\mathrm{deg}) \\
\text { Desired }\end{array}$ & $\begin{array}{c}\theta(\mathrm{deg}) \\
\text { Desired }\end{array}$ & $\begin{array}{c}\phi(\mathrm{deg}) \\
\text { Obtained }\end{array}$ & $\begin{array}{c}\theta(\mathrm{deg}) \\
\text { Obtained }\end{array}$ & $\begin{array}{c}\text { PSLL } \\
(\mathrm{dB}) \\
\text { URA }\end{array}$ & $\begin{array}{c}\text { PSLL } \\
(\mathrm{dB}) \\
\text { Sparse }\end{array}$ \\
\hline 0 & 0 & 0 & 0 & -13.58 & -12.69 \\
\hline 0 & 45 & 0 & 45 & -11.64 & -11.59 \\
\hline 45 & 0 & 46 & 0 & -11.48 & -12.48 \\
\hline 45 & -45 & 45 & -45 & -10.38 & -9.89 \\
\hline 20 & 30 & 20 & 30 & -12.27 & -12.98 \\
\hline-20 & 30 & -20 & 30 & -12.27 & -12.02 \\
\hline-35 & -45 & -35 & -45 & -11.00 & -11.23 \\
\hline 10 & -45 & 10 & -45 & -11.60 & -11.73 \\
\hline-10 & -40 & -10 & -40 & -11.85 & -11.75 \\
\hline-30 & 40 & -30 & 40 & -11.58 & -11.98 \\
\hline
\end{tabular}

It is observed that the values of $\phi$ and $\theta$ obtained are almost the same as the required values of the parameters. This observation validates the accuracy of the ANN model to predict the values of $\delta_{\mathrm{AZ}}$ and $\delta_{\mathrm{EL}}$. It also validates how the problem is formulated where the excitation weights of the sparse array are obtained from the Hadamard product of the weight matrix, $\mathrm{W}$ of the URA, and the binary matrix $\mathrm{B}$.

The PSLL values are obtained from the normalized radiation pattern of the arrays. It is observed that the PSLL values of the sparse array are close to that of the original URA. Thus, there is no significant increase in the side-lobe level due to thinning the array. Fig. 8 shows the normalized radiation pattern of the antenna at $\phi=30$ degree and $\theta=40$ degree. For easier comparison, the values where the values of the radiation pattern are less than $-60 \mathrm{~dB}$ are flattened.

From these figures, it is observed that the width and positions of the main lobe are identical for the URA and the sparse array. Although the sparse array has a larger number of side lobes, the values of these lobes are very small. Since the objective of the optimization problem was to suppress the PSLL only, the other side lobes are not significantly reduced.

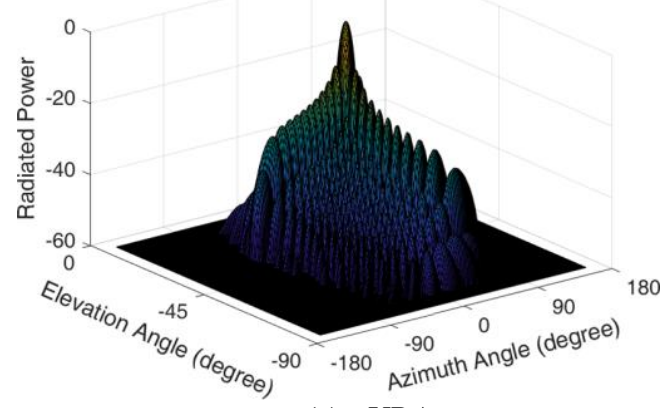

(a) URA

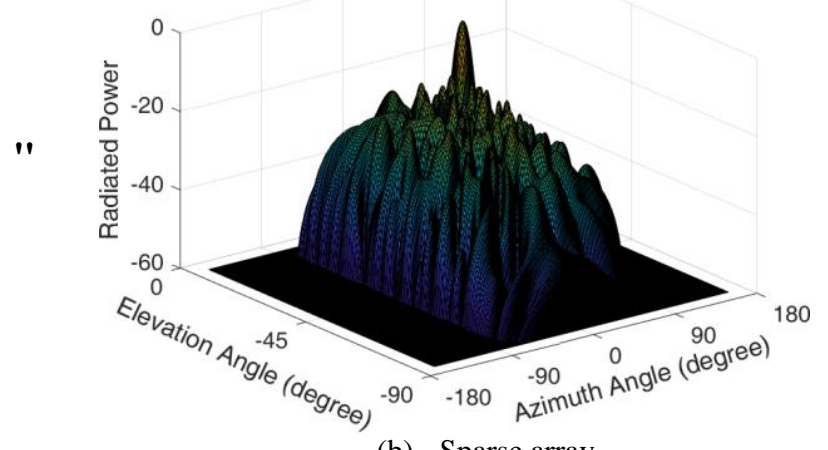

(b) Sparse array

Fig. 8. The radiation pattern of the (a) URA and (b) the synthesized sparse array for $\phi=30$ degree and $\theta=40$ degree

It is not possible to include all the radiation patterns in this paper. Therefore, the overall PSLL values of the URA and the proposed sparse array are compared in a 3D surface plot shown in Fig. 9. Here the values of $\phi$ and $\theta$ are tuned over the range of -45 degree to +45 degree. It is observed that only at these two extreme points, the PSLL value of the sparse array is slightly higher than that of the original URA. As the $\phi$ and $\theta$ approach $(0,0)$, the values of the PSLL of the URA and sparse array become almost the same. 


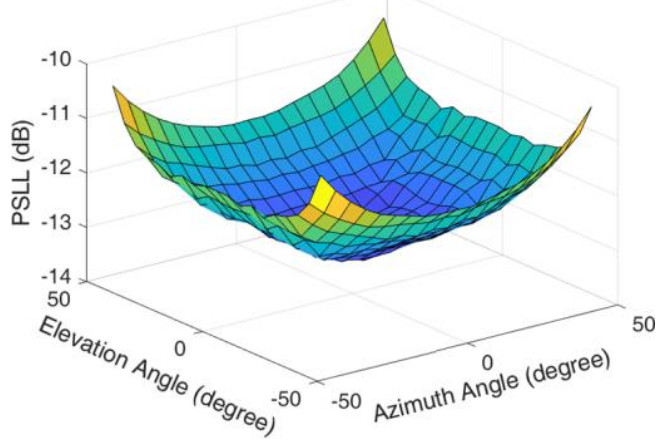

(a) URA

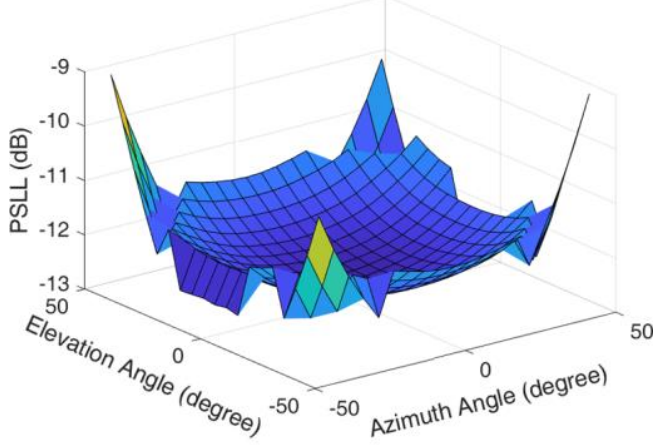

(b) Sparse array

Fig. 9. Variation of PSLL with the direction of the main lobe for (a) URA (b) the sparse array

\section{१ए\&RQFOMRQ}

A technique for synthesizing a sparse array from a $16 \times 16$ URA is presented. The excitation weight matrix, $\mathrm{W}$ of the URA are estimated using an ANN model from the desired scan-angle. The PSO is used for obtaining a binary matrix B, such that the Hadamard product of $\mathrm{B}$ and $\mathrm{W}$ yields the excitation weights of the sparse antenna array. The experimental results show that the desired scan-angles of the sparse array are accurately obtained using this technique.

The PSLL of the URA and the sparse array are compared for all possible scan-angles in a range of -45 degree to +45 degree for both the elevation plane and the azimuthal plane. It is observed that the PSLL of the synthesized sparse array is almost the same as that of the URA except at the extreme ends of the scanning range.

The overall scan angle of the proposed antenna array is 90 degree for both the azimuth plane and the elevation plane. The array comprises cosine antenna elements that represent printed antennas used in 5G millimeter-wave wireless communication. Thus, the proposed sparse array has possible applications in $5 \mathrm{G}$ wireless communication and radar systems.

\section{HHHQFHM}

[1] W. Hong, K. Baek and S. Ko, "Millimeter-Wave 5G Antennas for Smartphones: Overview and Experimental Demonstration," in IEEE
Transactions on Antennas and Propagation, vol. 65, no. 12, pp. 62506261, Dec. 2017, doi: 10.1109/TAP.2017.2740963.

[2] Z. Chen and Y. P. Zhang, "FR4 PCB grid array antenna for millimeter-wave 5G mobile communications," 2013 IEEE MTT-S International Microwave Workshop Series on RF and Wireless Technologies for Biomedical and Healthcare Applications (IMWSBIO), 2013, pp. 1-3, doi: 10.1109/IMWS-BIO.2013.6756214.

[3] R. J. Mailloux, Phased Array Antenna Handbook, Artech House, 2017.

[4] O. G. Vendik and Y. V. Yegorov, "The first phased-array antennas in Russia: 1955-1960," in IEEE Antennas and Propagation Magazine, vol. 42, no. 4, pp. 46-52, Aug. 2000, doi: 10.1109/74.868052.

[5] R. Munson, "Conformal microstrip antennas and microstrip phased arrays," in IEEE Transactions on Antennas and Propagation, vol. 22, no. 1, pp. 74-78, January 1974, doi: 10.1109/TAP.1974.1140723.

[6] A. H. Naqvi, and S. Lim, "Review of Recent Phased Arrays for Millimeter-Wave Wireless Communication", Sensors (Basel). Vol. 18, No. 10, Oct. 2018, doi: 10.3390/s18103194.

[7] C. I. Coman, I. E. Lager and L. P. Ligthart, "Design considerations in sparse array antennas," 2006 European Radar Conference, 2006, pp. 72-75, doi: 10.1109/EURAD.2006.280276.

[8] R. Jain, and G. S. Mani, "Solving "Antenna Array Thinning Problem", Using Genetic Algorithm”, Applied Computational Intelligence and Soft Computing, Vol. 2012, Article ID 946398, Apr 2012, doi: 10.1155/2012/946398.

[9] M. A. Zaman, and M. A. Matin, "Nonuniformly Spaced Linear Antenna Array Design Using Firefly Algorithm", International Journal of Microwave Science and Technology, Vol. 2012, Article ID 256759, Apr 2012, doi: doi.org/10.1155/2012/256759.

[10] E. Sandi, F. Y. Zulkifli, and E. T. Rahardjo, "A Hybrid Technique Using Combinatorial Cyclic Difference Sets and Binomial Amplitude Tapering for Linear Sparse Array Antenna Design", AEM, vol. 5, no. 3, pp. 73-79, Dec. 2016, doi: 10.7716/aem.v5i3.432

[11] K. Y. Reddy, R. B. Kumar, M. Jijenth, K. K. Suman, V. S. Gangwar and A. K. Singh, "Synthesis of randomly spaced planar antenna array with low peak side lobe level (PSLL) using Modified Genetic Algorithm," 2017 IEEE International Conference on Antenna Innovations \& Modern Technologies for Ground, Aircraft and Satellite Applications (iAIM), 2017, pp. 1-4, doi: 10.1109/IAIM.2017.8402567.

[12] H. Li, F. He, Y. Chen, and J. Luo, "Multi-objective self-organizing optimization for constrained sparse array synthesis," Swarm and Evolutionary Computation, vol. 58, p. 100743, Nov. 2020, doi: 10.1016/j.swevo.2020.100743.

[13] P. Gu, G. Wang, Z. Fan and R. Chen, "An Efficient Approach for the Synthesis of Large Sparse Planar Array," in IEEE Transactions on Antennas and Propagation, vol. 67, no. 12, pp. 7320-7330, Dec. 2019, doi: 10.1109/TAP.2019.2931959.

[14] S. Koziel and S. Ogurtsov, Antenna Design by Simulation-Driven Optimization. Springer International Publishing, 2014. doi: 10.1007/978-3-319-04367-8.

[15] F. Burden and D. Winkler, "Bayesian Regularization of Neural Networks," in Methods in Molecular Biology ${ }^{\mathrm{TM}}$, Humana Press, 2008, pp. 23-42. doi: 10.1007/978-1-60327-101-1_3.

[16] K. Sarmah, S. Goswami, and S. Baruah, "Surrogate Model Assisted Design of CSRR Structure using Genetic Algorithm for Microstrip Antenna Application," RADIOENGINEERING, vol. 29, no. 1, pp. 117-124, Apr. 2020, doi: 10.13164/re.2020.0117.

[17] S. M. Almufti, A. Yahya Zebari, and H. Khalid Omer, "A comparative study of particle swarm optimization and genetic algorithm," JACST, vol. 8, no. 2, p. 40, Oct. 2019, doi: 10.14419/jacst.v8i2.29401.

\section{Creative Commons Attribution License 4.0 (Attribution 4.0 International, CC BY 4.0)}

This article is published under the terms of the Creative Commons Attribution License 4.0 https://creativecommons.org/licenses/by/4.0/deed.en_US 